\title{
The Pomeron spin-flip and its measurements
}

\author{
M. Krelina* \\ Universidad Tecnica Federico Santa Maria, Casilla 110, Valparaiso, Chile; Czech Technical \\ University in Prague, FNSPE, Brehova 7, 11519 Prague, Czech Republic \\ E-mail: michal.krelina@usm.cl
}

\section{B.Z. Kopeliovich}

Universidad Tecnica Federico Santa Maria \& CCTVAL, Casilla 110, Valparaiso, Chile

E-mail: boris.kopeliovich@usm.cl

\begin{abstract}
We investigate the spin-flip component of the Pomeron using the single spin asymmetry, $A_{N}(t)$ in polarized elastic $p p$ scattering, arising from Coulomb-nuclear interference (CNI) in smallangle elastic scattering. The previous analyses have missed the absorptive corrections to the Coulomb amplitude of $p p$ elastic scattering, which plays an important role. Namely, interference of ultra-peripheral and central collisions leads to significant changes in $A_{N}(t)$. We found that the absorptive corrections considerably affect the results of the analysis of the single spin asymmetry data from STAR data for $A_{N}(t)$ at $\sqrt{s}=200 \mathrm{GeV}$. At such high energy, the Reggeon contribution is expected to be small so that one can study the Pomeron spin-flip component.
\end{abstract}

23rd International Spin Physics Symposium - SPIN2018

10-14 September, 2018

Ferrara, Italy

${ }^{*}$ Speaker. 


\section{Introduction}

Pomeron is the dominant Regge exchange in the high energy elastic hadronic amplitude. However, there is no consensus so far on the magnitude of the spin-flip part of the Pomeron, which can be studied by measuring the single spin asymmetry of polarized $p p$ elastic scattering in the Coulomb-nuclear interference (CNI) region [1]. The dominant contribution to the single-spin asymmetry $A_{N}(t)$ comes from the interference of the hadronic non-flip amplitude and Coulomb spin-flip term $[2,3]$. This topic is becoming more actual with the new results on $A_{N}(t)$ of protonproton elastic scattering at different collision energies from HJET measurement $[4,5,6]$ and STAR experiment [7] at RHIC.

The magnitude of spin-flip hadronic interaction can be studied in single spin asymmetry by introducing a ratio of spin-flip to the imaginary part of the non-flip hadronic elastic amplitudes, $r_{5}$ [8],

$$
r_{5} \equiv \frac{m_{N}}{\sqrt{-t}} \frac{\phi_{5}^{h}}{\operatorname{Im} \phi_{+}^{h}},
$$

where $\phi_{+}^{h}$ and $\phi_{5}^{h}$ are hadronic non-flip and spin-flip amplitudes, respectively. $m_{N}$ is the nucleon mass, and $t=-q^{2}$ is the momentum transferred. This parameter can be extracted from the difference between experimental data and theoretical predictions with the spin-flip interaction of Coulomb origin only.

The parameter $r_{5}$ is not a pure observable for the spin-flip interaction of Pomeron. It is true for very high energies, such as at STAR, i.e., $\sqrt{s}=200 \mathrm{GeV}$. However, the lower energies of HJET experiment, $E_{\text {lab }}=100$ and $255 \mathrm{GeV}$ equivalent to $\sqrt{s}=13.76$ and $21.92 \mathrm{GeV}$, might be not sufficiently high to eliminate the Reggeon contributions. Note, the Regge phenomenology can describe $p p$ interactions at low energies via exchange of $\omega, f, \rho$ and $a_{2}$ Reggeons, where the last two are the iso-vector Reggeons, which are known to be predominantly spin-flip.

The contribution of Reggeons will be studied in detail in the upcoming paper [9]. In this short report, we want to focus on the other omitted effect, the absorptive corrections to the Coulomb amplitude.

\section{Single spin asymmetry in elastic $p p$ scattering}

Proton-proton elastic scattering can be described by five independent amplitudes, [8], where every amplitude is a sum of the hadronic $(h)$ and Coulomb $(\mathrm{em})$ contributions,

$$
\phi_{1}=\phi_{1}^{h}+\phi_{1}^{e m} .
$$

The single spin asymmetry, $A_{N}$, and the differential cross section read

$$
\begin{aligned}
A_{N} \frac{d \sigma}{d t} & =-\frac{4 \pi}{s^{2}} \operatorname{Im}\left\{\left(\phi_{1}+\phi_{2}+\phi_{3}-\phi_{4}\right) \phi_{5}^{*}\right\}, \\
\frac{d \sigma}{d t} & =\frac{2 \pi}{s^{2}}\left\{\left|\phi_{1}\right|^{2}+\left|\phi_{2}\right|^{2}+\left|\phi_{3}\right|^{2}+\left|\phi_{4}\right|^{2}+4\left|\phi_{5}\right|^{2}\right\} .
\end{aligned}
$$

In particular, the Coulomb amplitudes read,

$$
\phi_{1}^{e m}=\phi_{3}^{e m}=-\frac{\alpha_{e m}}{|t|} s G^{2}(t) e^{i \delta_{p p}},
$$




$$
\begin{aligned}
& \phi_{2}^{e m}=\phi_{4}^{e m} \approx 0, \\
& \phi_{5}^{e m}=-\frac{\alpha_{e m}\left(\mu_{N}-1\right)}{2 m_{N} \sqrt{-t}} s G^{2}(t) e^{i \delta_{p p}},
\end{aligned}
$$

where $\mu_{N}$ is the anomalous magnetic moment of the proton, $\delta_{p p}$ is the Coulomb phase [10], and the electromagnetic form factor $G^{2}(t)$ is taken in the form

$$
G^{2}(t)=e^{-\frac{1}{3}\left\langle r_{E M}^{2}\right\rangle|t|},
$$

where $\left\langle r_{E M}^{2}\right\rangle$ is the proton mean charge radius squared.

\section{Absorptive corrections}

The proton electromagnetic formfactor has been measured in electron-proton scattering, free of initial/final state strong interactions between the colliding particles, while in proton-proton scattering the absorptive corrections may substantially reduce the partial elastic amplitude at small impact parameters (see Fig. 1, right). Such corrections were evaluated for proton-nucleus collisions [11].
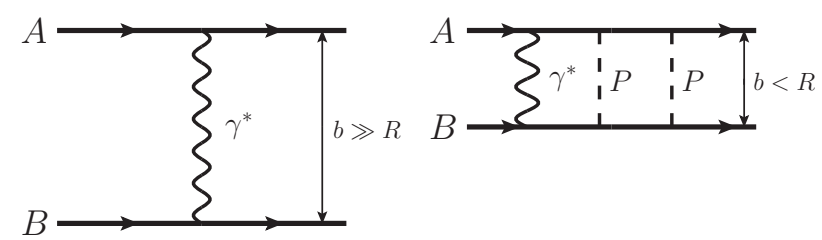

Figure 1: At large interaction distances only the Coulomb interaction applies. Nevertheless, when at small impact parameters, besides the photon exchange, there is a high probability of the additional Pomeron exchanges.

Notice that absorptive corrections frequently introduced on the probabilistic level as a rapidity gap survival probability factor in the cross section. However, we rely on the correct quantummechanical treatment and introduce the absorptive corrections to the amplitude, as is illustrated in Fig. 1, and do it as follows. First, we Fourier transform the amplitudes (2.4) and (2.6) to impact parameter space. Then the $b$-dependent partial amplitude is corrected for absorption as

$$
\phi_{i}^{e m}(b) \Rightarrow \phi_{i}^{e m}(b) S(b),
$$

where the absorption correction reads

$$
S(b) \equiv 1-\operatorname{Im} f_{e l}(b) .
$$

$f_{e l}(b)$ is the partial elastic amplitude for hadronic interaction in the impact parameter space. Assuming Gaussian $b$-dependence, one gets,

$$
\operatorname{Im} f_{e l}(b)=\frac{\sigma_{t o t}^{p p}}{4 \pi B} e^{-\frac{b^{2}}{2 B}}
$$

where $\sigma_{t o t}^{p p}$ is the total proton-proton cross section and $B$ is the slope parameter of the differential cross section.

The last step is the application of the inverse Fourier transformation back to the momentum representation. 


\section{Results and conclusions}

Table 1 presents the results of the fit of Eqs. (2.2)-(2.3 to the experimental data $[4,5,6]$ without absorptive corrections. The fitted parameter $r_{5}$ turns out to be consistent with zero. Since at such high energy the Reggeons are not expected, one would conclude that the Pomeron conserves helicity. The quality of the description of the STAR data with zero $r_{5}$ and no absorptive correction is shown in Fig. 2 by the dashed-dotted curve.

\begin{tabular}{|l|l|l|l|l|l|}
\hline Energy & note & $\operatorname{Re} r_{5}$ & $\operatorname{Im} r_{5}$ & $\chi^{2}$ & NDf \\
\hline 200 & STAR & $0.0021 \pm 0.0031$ & $0.0088 \pm 0.0524$ & 0.633268 & 3 \\
21.92 & HJET & $-0.0074 \pm 0.0012$ & $0.0152 \pm 0.0060$ & 18.8155 & 74 \\
13.76 & HJET & $-0.0176 \pm 0.0021$ & $-0.0013 \pm 0.0068$ & 10.3155 & 86 \\
\hline
\end{tabular}

Table 1: Fit of $r_{5}$ without absorptive correction.

However, the introduction of absorptive corrections leads to drastic changes in the fit. The dashed curve demonstrates sensitivity to the absorptive corrections, which cause the difference from the above mentioned dashed-dotted curve. The new fit including absorption is presented in Table 2, and is depicted by the solid red curve in Fig. 2.

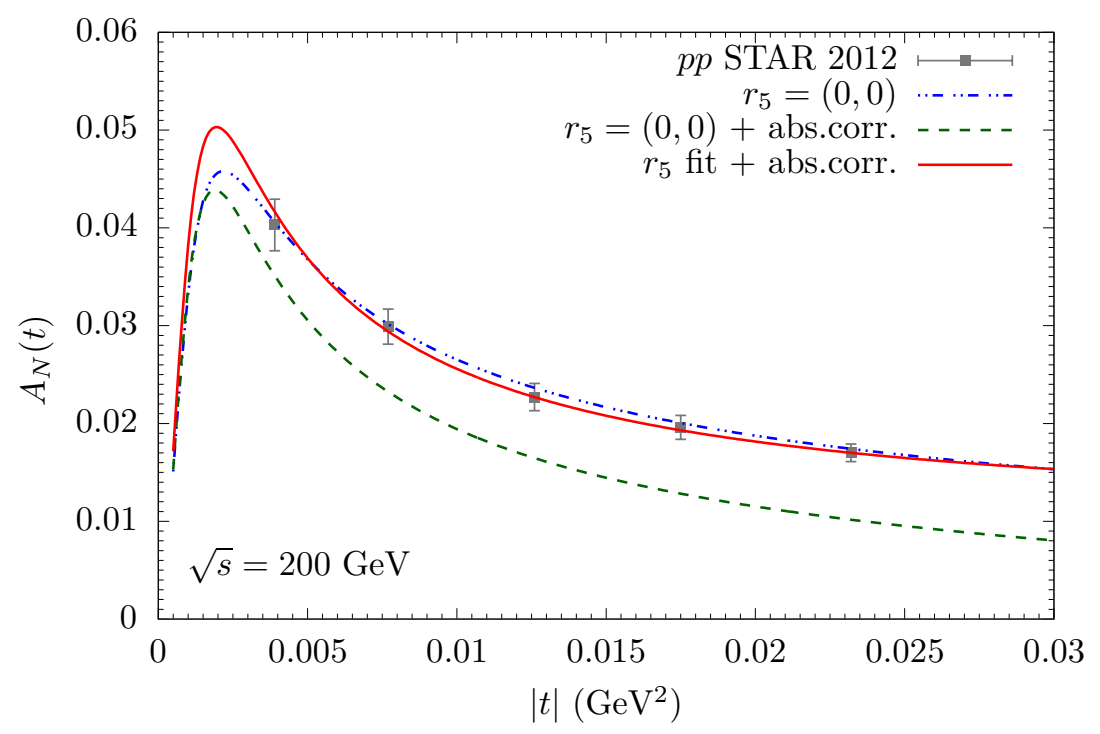

Figure 2: Data vs calculation with and without absorption correction.

\begin{tabular}{|l|l|l|l|l|l|}
\hline Energy $\sqrt{s}$ & note & $\operatorname{Re} r_{5}$ & $\operatorname{Im} r_{5}$ & $\chi^{2}$ & NDf \\
\hline 200 & STAR & $-0.0363 \pm 0.0080$ & $-0.0843 \pm 0.0524$ & 1.27379 & 3 \\
21.92 & HJET & $-0.0330 \pm 0.0007$ & $0.0024 \pm 0.0058$ & 34.3497 & 74 \\
13.76 & HJET & $-0.0472 \pm 0.0014$ & $0.0209 \pm 0.0063$ & 26.5716 & 86 \\
\hline
\end{tabular}

Table 2: Fit of $r_{5}$ with absorptive correction.

Summarizing, we demonstrated the importance of the absorptive correction to the Coulomb amplitude. These corrections significantly affect the fitted value of $r_{5}$, which can be attributed to 
the Pomeron. However, in the case of HJET measurements at lower energies, Secondary Reggeons become important, and one cannot treat the resulting $r_{5}$ as a measure of the Pomeron spin-flip. The contribution of Reggeons must be taken into account as was discussed in [12].

\section{Acknowledgement}

The work of Michal Krelina was supported in part by Fondecyt grant Postdoctorado $\mathrm{N}^{\circ} 3180085$, by the grant 17-04505S of the Czech Science Foundation (GACR) and partly this work has been performed in the framework of COST Action CA15213 THOR. Boris Kopeliovich was partially supported by Fondecyt grant No. 1170319, by Proyecto Basal FB 0821, and by Conicyt grant PIA ACT1406.

\section{References}

[1] B. Z. Kopeliovich and B. G. Zakharov, Phys. Lett. B 226 (1989) 156.

[2] B. Z. Kopeliovich and L. I. Lapidus, Yad. Fiz. 19 (1974) 340.

[3] B. Z. Kopeliovich, arXiv:hep-ph/9801414, (1998).

[4] A. Poblaguev at al., in preparation (proceeding of SPIN2016).

[5] A. Poblaguev at al., in preparation (proceeding of SPIN2018).

[6] A. Poblaguev et al., PoS PSTP 2017, 022 (2018).

[7] L. Adamczyk et al. [STAR Collaboration], Phys. Lett. B 719, 62 (2013).

[8] N. H. Buttimore at al., Phys.Rev.D59, (1999) 114010.

[9] B. Z. Kopeliovich and M. Krelina, in preparation.

[10] B. Z. Kopeliovich and A. V. Tarasov, Phys. Lett. B 497, 44 (2001)

[11] M. Krelina and B. Z. Kopeliovich, arXiv:1711.01969 [hep-ph].

[12] B. Z. Kopeliovich, "Pomeron spin-flip", a talk at the CFNS workshop on Forward Physics, Stony Brook, Oct. 17-19, 2018. https://indico.bnl.gov/event/4737/timetable/ 